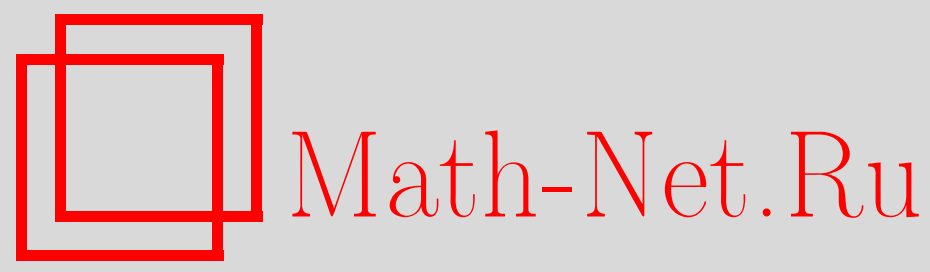

А. В. Филиновский, О непрерывности спектра задачи Неймана для эллиптических операторов второго порядка в расширяющихся областях, Матем. заметки, 1997, том 61, выпуск $3,471-475$

DOI: https://doi.org/10.4213/mzm1526

Использование Общероссийского математического портала Math-Net.Ru подразумевает, что вы прочитали и согласны с пользовательским соглашением http://www . mathnet.ru/rus/agreement

Параметры загрузки:

IP : 3.80 .181 .102

26 апреля 2023 г., 12:26:11

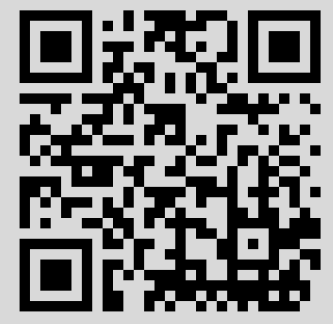




\section{О НЕПРЕРЫВНОСТИ СПЕКТРА ЗАДАЧИ НЕЙМАНА ДЛЯ ЭЛЛИПТИЧЕСКИХ ОПЕРАТОРОВ ВТОРОГО ПОРЯДКА В РАСШИРЯЮШИХСЯ ОБЛАСТЯХ}

\section{А.В. Филиновский}

Пусть $\Omega \subset \mathbb{R}^{n}, n \geqslant 2,-$ неограниченная область с границей $Г$. Предположим, что для любого $R>0$ часть гранишы $\Gamma \cap\{|x|<R\}$ состоит из конечного числа поверхностей класса $C^{2}$. Рассмотрим дифференциальное выражение

$$
l v=-\sum_{j=1}^{n} \frac{\partial}{\partial x_{j}}\left(a(x) \frac{\partial v}{\partial x_{j}}\right), \quad x \in \Omega
$$

где функция $a(x)$ вешественнозначна, непрерьвно дифференцируема в $\bar{\Omega}$ и удовлетворяет неравенствам

$$
0<\gamma_{1} \leqslant a(x) \leqslant \gamma_{2}, \quad x \in \Omega
$$

Обозначим через $L_{0}$ минимальньй оператор, порожденный в $L_{2}(\Omega)$ дифференциальным выражением (1), $L$ - самосопряженное расширение $L_{0}$, соответствуюшее граничному условию

$$
\left.\frac{\partial v}{\partial \nu}\right|_{\Gamma}=0
$$

где $\nu$ - единичньй вектор внешней по отношению к $\Omega$ нормали к $\Gamma$. Из самосопряженности и положительности $L$ следует, что $\sigma(L)=\sigma_{p}(L) \cup \sigma_{c}(L) \subset[0,+\infty)$, где $\sigma_{p}(L)-$ точечньй, а $\sigma_{c}(L)$ - непрерьвньй спектр оператора $L$.

Сформулируем известные условия непрерывности спектра оператора $L$. В случае компактной $\Gamma$ для $n \geqslant 2$ и $a(x) \equiv a_{\infty}=$ const в [1] доказано, что $(0,+\infty) \subset \sigma_{c}(L)$. Этот результат распространен в работе [2] на функции $a(x)$, удовлетворяюшие условиям

$$
\begin{gathered}
a(x)=a_{\infty}+O\left(|x|^{-1-\varepsilon}\right), \quad|\nabla a(x)|=O\left(|x|^{-1-\varepsilon}\right), \quad\left|\nabla^{2} a(x)\right|=O\left(|x|^{-2-\varepsilon}\right), \\
|x| \rightarrow \infty, \quad \varepsilon>0,
\end{gathered}
$$

а в [3] условия на $a(x)$ ослаблены

$$
a(x)=a_{\infty}+o(1), \quad|\nabla a(x)|=O\left(|x|^{-1-\varepsilon}\right), \quad|x| \rightarrow \infty, \quad \varepsilon>0 .
$$

В работе [4] непрерьвность спектра оператора $L$ на полуоси $(0,+\infty)$ (Г компактна) установлена при

$$
|\nabla a(x)|=o\left(|x|^{-1}\right), \quad\left|\nabla^{2} a(x)\right|=o\left(|x|^{-2}\right), \quad|x| \rightarrow \infty .
$$

В настоящей работе изучаются классы областей $\Omega$ с бесконечными границами, для которых спектр оператора $L$ непрерывен. Пусть $0<\alpha_{1} \leqslant \alpha_{2} \leqslant \ldots \leqslant \alpha_{n}=1$ и $\varphi(x)=\alpha_{1} x_{1}^{2}+\cdots+\alpha_{n} x_{n}^{2}$. 
ОПРЕДЕЛЕНИЕ 1 . Область $\Omega$ называется областью класса $D_{\varphi}^{N}$, если

$$
(\nu, \nabla \varphi(x))=0, \quad x \in \Gamma .
$$

Например, область $\Omega=\left\{x: C_{1} x_{2}^{\alpha}<x_{1}<C_{2} x_{2}^{\alpha}, x_{2}>0\right\} \subset \mathbb{R}^{2}, 0<C_{1}<C_{2}$, принадлежит классу $D_{\varphi}^{N}$ с $\varphi(x)=\alpha x_{1}^{2}+x_{2}^{2}$. При $\alpha_{1}=\cdots=\alpha_{n}=1$ область $\Omega \in D_{\varphi}^{N}$ представляет собой внутренность конуса с вершиной в начале координат. Из (4) следует, что граница области $\Omega \in D_{\varphi}^{N}, \Omega \neq \mathbb{R}^{n}$, бесконечна.

Близкие к $D_{\varphi}^{N}$ классы областей, связанные с задачей Дирихле, рассматривались в работе [5].

Обозначим

$$
\rho=\sqrt{\varphi(x)}, \quad a_{\rho}=\frac{(\nabla a, \nabla \varphi)}{|\nabla \varphi|}, \quad v_{\rho}=\frac{(\nabla v, \nabla \varphi)}{|\nabla \varphi|} .
$$

TEOPEMA 1. Пусть $n \geqslant 2, \alpha_{1}=1, \Omega \in D_{\varphi}^{N} u$

$$
-\frac{n-1}{\rho+1} \gamma_{1}<a_{\rho}<\frac{2 \alpha_{1}}{\rho+1} \gamma_{1}, \quad x \in \Omega .
$$

Тогда $\sigma_{p}(L)=\varnothing$.

Доказательство теоремы 1 следует из лемм 2 и 3.

Лемма 1. Пусть $n \geqslant 2, \Omega \in D_{\varphi}^{N}$ u $v(x, \lambda) \not \equiv 0-$ решение задачи

$$
\begin{gathered}
l v-\lambda v=0, \quad \lambda>0, \quad x \in \Omega, \\
\left.\frac{\partial v}{\partial \nu}\right|_{\Gamma}=0,
\end{gathered}
$$

$u$

$$
a_{\rho}<\frac{2 \alpha_{1}}{\rho+1} \gamma_{1}, \quad x \in \Omega .
$$

Тогда существуют такие числа $C_{3}>0$ и $R_{1}>0$, что

$$
\int_{S_{R}^{\varphi}}\left(\left|v_{\rho}\right|^{2}+|v|^{2}\right) d s \geqslant \frac{C_{3}}{R}, \quad R \geqslant R_{1}, \quad S_{R}^{\varphi}=\Omega \cap\{\rho=R\} .
$$

ДокАЗАТЕЛЬСтво. Достаточно доказать (9) для вещественной или мнимой частей $v(x, \lambda)$, которые также удовлетворяют $(6),(7)$. Поэтому функцию $v(x, \lambda)$ будем считать вешественнозначной. Умножим уравнение $(6)$ на $2(\nabla v, \nabla \varphi)$ и проинтегрируем по $\Omega_{R}^{\varphi}=\Omega \cap\{\rho<R\}$ с учетом (7). Получим равенство

$$
\begin{gathered}
\int_{S_{R}^{\varphi}} a|\nabla \varphi|\left(2 v_{\rho}^{2}-|\nabla v|^{2}+\frac{\lambda}{a} v^{2}+\frac{\Delta \varphi}{|\nabla \varphi|} v v_{\rho}\right) d s \\
=\int_{\Gamma_{R}^{\varphi}}(\nu, \nabla \varphi)\left(a|\nabla v|^{2}-\lambda v^{2}\right) d s+\int_{\Omega_{R}^{\varphi}}\left(2 a \sum_{j=1}^{n} \varphi_{x_{j} x_{j}} v_{x_{j}}^{2}-(\nabla a, \nabla \varphi)|\nabla v|^{2}\right) d x \\
\Gamma_{R}^{\varphi}=\Gamma \cap\{\rho<R\}
\end{gathered}
$$


Из (4), (8) и (10) следует, что при $R \geqslant R_{1}>0$

$$
\begin{aligned}
0 & \leqslant \int_{\Omega_{R_{1}}^{\varphi}}|\nabla \varphi|\left(\frac{2 \alpha_{1} \gamma_{1}}{\rho+1}-a_{\rho}\right)|\nabla v|^{2} d x \leqslant \int_{\Omega_{R}^{\varphi}}|\nabla \varphi|\left(\frac{2 \alpha_{1} \gamma_{1}}{\rho+1}-a_{\rho}\right)|\nabla v|^{2} d x \\
& \leqslant \int_{\Omega_{R}^{\varphi}}\left(4 a \sum_{j=1}^{n} \alpha_{j} v_{x_{j}}^{2}-(\nabla a, \nabla \varphi)|\nabla v|^{2}\right) d x \\
& =\int_{S_{R}^{\varphi}} a|\nabla \varphi|\left(2 v_{\rho}^{2}-|\nabla v|^{2}+\frac{\lambda}{a} v^{2}+\frac{\Delta \varphi}{|\nabla \varphi|} v v_{\rho}\right) d s \\
& \leqslant 2 R \gamma_{2} \int_{S_{R}^{\varphi}}\left(\left(2+\frac{\Delta \varphi}{2|\nabla \varphi|}\right) v_{\rho}^{2}+\left(\frac{\lambda}{\gamma_{1}}+\frac{\Delta \varphi}{2|\nabla \varphi|}\right) v^{2}\right) d s \leqslant C_{4} R \int_{S_{R}^{\varphi}}\left(v_{\rho}^{2}+v^{2}\right) d s .
\end{aligned}
$$

Если

$$
\int_{\Omega_{R_{1}}^{\varphi}}|\nabla v|^{2} d x=0 \quad \text { для всех } R_{1}>0,
$$

то $v \equiv C_{5} \neq 0$ и неравенство (9) выполнено. В случае

$$
\int_{\Omega_{R_{1}}^{\varphi}}|\nabla v|^{2} d x>0
$$

при некотором $R_{1}>0$ неравенство (9) следует из (11).

Лемма 2. Пусть $n \geqslant 2, \alpha_{1}=1, \Omega \in D_{\varphi}^{N}, v(x, \lambda) \not \equiv 0-$ решение задачи (6), (7), $\lambda>0$, функиия $a(x)$ удовлетворяет условию (5). Тогда существуют число $C_{6}>0$ $u$ последовательность $R_{m} \rightarrow \infty, m \rightarrow \infty$, такие, что

$$
\int_{\Omega_{R_{m}}^{\varphi}}|v|^{2} d x \geqslant C_{6} \ln R_{m}, \quad m=1,2, \ldots
$$

ДокАЗАТЕЛЬСТво. Как и при доказательстве леммы 1, будем считать функцию $v(x, \lambda)$ вешественнозначной. Пусть

$$
J_{\varphi}(R)=\int_{S_{R}^{\varphi}} a v^{2} d s
$$

Возможны два случая:

а) $J_{\varphi}^{\prime}(R) \geqslant 0$ для всех $R \geqslant R_{2}>0$;

б) сушествует последовательность $R_{m} \rightarrow \infty, m \rightarrow \infty$, такая, что $J_{\varphi}^{\prime}\left(R_{m}\right)<0$.

В случае а) при всех $R>R_{2}>0$ и некотором $C_{6}>0 J(R)>C_{6}$, а потому

$$
\int_{\Omega_{R}^{\varphi}} v^{2} d x \geqslant \int_{R_{2}}^{R} J(\rho) d \rho \geqslant C_{6}\left(R-R_{2}\right), \quad R \geqslant R_{2},
$$

откуда следует неравенство (12).

В случае б) из (4), (6), (7) и равенства

$$
\begin{gathered}
J_{\varphi}^{\prime}(R)=\frac{n-1}{R} J_{\varphi}(R)+\int_{S_{R}^{\varphi}} a_{\rho} v^{2} d s+2 \int_{S_{R}^{\varphi}} a v v_{\rho} d s-\int_{L_{R}^{\varphi}} \operatorname{ctg}(\nu, \nabla \varphi) a v^{2} d l, \\
R>0, \quad L_{R}^{\varphi}=\Gamma \cap\{\rho=R\},
\end{gathered}
$$


следует, что

$$
\begin{aligned}
\lambda \int_{\Omega_{R_{m}}^{\varphi}} v^{2} d x & =\int_{\Omega_{R_{m}}^{\varphi}} a|\nabla v|^{2} d x-\int_{S_{R_{m}}^{\varphi}} a v v_{\rho} d s \\
& =\int_{\Omega_{R_{m}}^{\varphi}} a|\nabla v|^{2} d x-\frac{1}{2} J_{\varphi}^{\prime}\left(R_{m}\right)+\frac{n-1}{2 R_{m}} \int_{S_{R_{m}}^{\varphi}} a v^{2} d s+\frac{1}{2} \int_{S_{R_{m}}^{\varphi}} a_{\rho} v^{2} d s \\
& \geqslant \int_{\Omega_{R_{m}}^{\varphi}} a|\nabla v|^{2} d x+\frac{1}{2} \int_{S_{R_{m}}^{\varphi}}\left(a_{\rho}+\frac{n-1}{\rho} \gamma_{1}\right) v^{2} d s \\
& \geqslant \int_{\Omega_{R_{m}}^{\varphi}} a|\nabla v|^{2} d x, \quad m=1,2, \ldots
\end{aligned}
$$

Поскольку из (9) вытекает оценка

$$
\int_{\Omega_{R}^{\varphi}}\left(|\nabla v|^{2}+v^{2}\right) d x \geqslant C_{7} \ln R, \quad R \geqslant R_{1},
$$

то, учитьвая (14), получаем требуемое неравенство (12).

ЛЕмма 3. Пусть $n \geqslant 2, v(x, 0) \not \equiv 0$ - решение задачи (6), (7), $\lambda=0$ и выполнено условие

$$
a_{\rho}>-\frac{n-1}{\rho+1} \gamma_{1}, \quad x \in \Omega .
$$

Тогда существуют такие числа $C_{8}>0 u R_{3}>0$, что

$$
\int_{\Omega_{R}^{\varphi}}|v|^{2} d x \geqslant C_{8} R^{2}, \quad R \geqslant R_{3} .
$$

ДоКАЗАТЕЛЬСТво. Как и в доказательствах лемм 1 и 2 , будем считать функцию $v$ вешественнозначной. Из (4), (6), (7) и (13) следует, что

$$
J_{\varphi}^{\prime}(R)=\frac{n-1}{R} J_{\varphi}(R)+\int_{S_{R}^{\varphi}} a_{\rho} v^{2} d s+2 \int_{\Omega_{R}^{\varphi}} a|\nabla v|^{2} d x .
$$

Если $v \equiv C_{9} \neq 0$, то

$$
\int_{\Omega_{R}^{\varphi}}|v|^{2} d x \geqslant C_{8} R^{n}, \quad R \geqslant R_{3}
$$

и неравенство (16) вьполнено. Если $v \neq C_{9}$, то из (15) и (17) следует, что

$$
\begin{gathered}
J_{\varphi}(R)=\int_{R_{3}}^{R} d \rho \int_{S_{\rho}^{\varphi}}\left(\frac{n-1}{\rho} a+a_{\rho}\right) v^{2} d s+2 \int_{R_{3}}^{R} d \rho \int_{\Omega_{\rho}^{\varphi}} a|\nabla v|^{2} d x \geqslant C_{9}\left(R-R_{4}\right), \\
R \geqslant R_{4}>0 .
\end{gathered}
$$

Поэтому

$$
\int_{\Omega_{R}^{\varphi}} v^{2} d x \geqslant C_{10} \int_{R_{3}}^{R} J_{\varphi}(\rho) d \rho \geqslant C_{8} R^{2}, \quad R \geqslant R_{5},
$$

и лемма 3 доказана.

Изучим плотность множества $\sigma(L)$ на $[0,+\infty)$. 
ОПРЕДЕЛЕНИЕ 2 [6]. Область $\Omega$ называется квазиконической, если она содержит шар любого радиуса.

$\mathrm{B}[6]$ для квазиконической $\Omega$ и $a(x) \equiv a_{\infty}$ установлено равенство $\sigma(L)=[0,+\infty)$.

ТЕОРема 2. Пусть $n \geqslant 2, \Omega \subset \mathbb{R}^{n}-$ квазиконическая область $и$

$$
a(x)=a_{\infty}+o(1), \quad|\nabla a(x)|=o(1), \quad|x| \rightarrow \infty .
$$

Тогда $\sigma(L)=[0,+\infty)$.

ДокАЗАТЕЛЬСТво. Пусть $\eta(x) \in \stackrel{\circ}{C}^{\infty}[-1,1],\|\eta\|_{C[-1,1]}=1,\|\eta\|_{L_{2}(-1,1)}=1$. Так как $\Omega$ квазиконическая, то сушествует последовательность непересекаюшихся шаров

$$
\begin{gathered}
B_{R_{m}}\left(x_{m}\right)=\left\{x:\left|x-x_{m}\right|<R_{m}\right\} \subset \Omega, \quad\left|x_{m}\right|=\left|\left(x_{1 m}, \ldots, x_{n m}\right)\right| \rightarrow \infty, \\
R_{m} \rightarrow \infty, \quad m \rightarrow \infty .
\end{gathered}
$$

Рассмотрим функции

$$
\begin{array}{r}
v_{m}(x)=R_{m}^{-n / 2} \eta\left(\frac{x_{1}-x_{1 m}}{R_{m}}\right) \eta\left(\frac{x_{2}-x_{2 m}}{R_{m}}\right) \cdots \eta\left(\frac{x_{n}-x_{n m}}{R_{m}}\right) e^{i \sqrt{\lambda / a_{\infty}} x_{1}}, \\
m=1,2, \ldots, \quad \lambda \geqslant 0,
\end{array}
$$

для которых $\operatorname{supp} v_{m}(x) \subset B_{R_{m}}\left(x_{m}\right),\left\|v_{m}(x)\right\|_{C\left(B_{R_{m}}\right)}=R_{m}^{-n / 2},\left\|v_{m}(x)\right\|_{L_{2}\left(B_{R_{m}}\right)}=1$ и $\left(v_{j}, v_{m}\right)_{L_{2}(\Omega)}=0, j \neq m$. Поскольку $v_{m}(x) \in D(L)$ и в силу (18) $\left\|l v_{m}-\lambda v_{m}\right\|_{L_{2}(\Omega)}=o(1)$, $m \rightarrow \infty$, из результатов работы [7] следует $\lambda \in \sigma(L)$.

Теорема 2 доказана.

Московский государственный технический университет

Поступило

им. Н.Э. Баумана

06.12 .96

\section{СПИСОК ЦИТИРОВАННОЙ ЛИТЕРАТУРЫ}

1. Rellich F. // Jahresber. Deutsch. Math.-Verein. 1943. V. 53. №1. P. 57-65. 2. Jäger W. // Math. Z. 1967. V. 102. №1. Р. 62-88. 3. Эйдус Д. М. // УМН. 1969. Т. 24. № 3. С. 91-156. 4. Vogelsang V. // J. Reine Angew. Math. 1983. V. 338. P. 109-120. 5. Филиновский А. В. // Матем. сб. 1996. Т. 187. №6. С. 131-160. 6. Глазман И. М. // Докл. АН СССР. 1952. Т. 87. № 2. С. 171-174. 7. Глазман И. М. // Докл. АН СССР. 1952. Т. 87. №1. С. 5-8. 